\title{
Sistemas de información: definiciones, usos y limitantes al caso de la producción ovina colombiana
}

\section{Information systems: definitions, applications and limiting of the colombian sheep production}

\author{
Sistemas de informação: definições, usos e limitações \\ para o caso da produção colombiana de ovelhas
}

Carlos A Vega-Pérez ${ }^{1}$, Henry A Grajales-Lombana ${ }^{2}$, Luz Alexandra Montoya Restrepo ${ }^{3}$

1 MVZ, Esp, MSc, Estudiante doctorado en producción animal UNAL FMVZ, Docente asistente UPTC Duitama

2 Zootecnista, MSc, PhD, Profesor Asociado, Departamento de Producción Animal, Facultad de Medicina Veterinaria y de Zootecnia, Universidad Nacional de Colombia.

3 Administradora de Empresas, MSc, PhD, Profesor Asociado, Facultad de Minas, Universidad Nacional de Colombia Sede Medellín.

Email: carlos.vega@uptc.edu.co

Recibido: 02 septiembre de $2015 \quad$ Aceptado: 20 de junio de 2017

\begin{abstract}
Resumen
El acceso rápido y eficiente a una información confiable y precisa permite adoptar una posición adecuada a la hora de tomar una decisión para solucionar un problema con un menor costo; la información es por lo tanto una sustancia figurativa, y se pueden nombrar ciertas posturas sobre ella: la posesión, la acumulación, exceso ("sobrecarga"), la desigualdad distributiva ("los que tienen y los que no"), la medición, la "comoditización", y así sucesivamente, antes de entender per se el uso de los sistemas de información. Es incuestionable el hecho de que mucha gente está derivando grandes ventajas de estas nuevas fuentes de conocimiento prestadas por redes y sistemas de información, en el marco de lo que ahora se convenciona llamar el sector cuaternario de la economía. Los datos, información y el conocimiento son eslabones de una cadena cíclica en la que el último eslabón realimenta la cadena, a la vez que el valor del recurso y la aportación e implicación humana son cada vez mayores. El objeto de la presente revisión es aportar al entendimiento de los sistemas de información y su orientación actual como herramienta para la mejora de la competitividad del sector ovino nacional.
\end{abstract}

Palabras clave: desarrollo tecnológico, Gestión, modelos, tradición.

\begin{abstract}
The fast and efficient access to reliable and accurate information allows adopting a suitable position when making a decision to troubleshoot a lower cost; information is thus a figurative substance, and can appoint certain positions on it: possession, accumulation, excess ("overload"), distributive inequality ("the haves and have-nots"), measurement, the "commoditization", and so on, before understanding per se the use of information systems. It is unquestionable that many people are deriving great advantages of these new sources of knowledge borrowed from networks and information systems, as part of what is now called the "quaternary sector of the economy". The data, information and knowledge are links in a cyclic chain where the last link in the chain fed, while the resource value and contribution and human involvement are
\end{abstract}


increasing. The purpose of this review is to provide an understanding of information systems and the current focus as a tool for improving the competitiveness of the Colombian domestic sheep industry.

Keywords: management, technology development, tradition, models

\begin{abstract}
Resumo
O acesso rápido e eficiente às informações confiáveis e precisas permitem adoptar uma posição adequada ao tomar uma decisão para solucionar um custo menor; informação é, portanto, uma substância figurativa, e pode designar certas posições sobre ele: a posse, a acumulação, o excesso ("sobrecarga"), a desigualdade distributiva ("os que têm e os que não têm"), medição, o "commoditization", e assim por diante, antes de compreender per se a utilização de sistemas de informação. É inquestionável que muitas pessoas estão a retirar grandes vantagens destas novas fontes de conhecimento emprestado de redes e sistemas de informação, como parte do que hoje é chamado de conven setor quaternário da economia. Os dados, informação e conhecimento são elos de uma cadeia cíclica, onde o último elo da cadeia alimentados, enquanto o valor de recursos e contribuição e envolvimento humano estão aumentando. O objetivo desta revisão é fornecer uma compreensão de sistemas de informação e o foco atual como uma ferramenta para melhorar a competitividade da indústria ovina doméstica.
\end{abstract}

Palavras chave: desenvolvimento tecnológico, gestão, modelos, tradição.

\section{Introducción}

Las recientes teorías económicas así como las evidencias acerca del crecimiento que se observa a partir de la década de los noventa (Stiroh, 1999) identifican dos características definitorias del fenómeno de la nueva economía: el auge de la globalización y la continua expansión de las tecnologías de la información. Estos dos aspectos no son síntomas de otros factores, son los factores que están propiciando el cambio y el crecimiento de la economía (Urquiza, 2007).

El acceso rápido y eficiente a una información confiable y precisa permite adoptar una posición adecuada a la hora de tomar una decisión para solucionar un problema con un menor costo (Espinosa, 2014) aun y cuando, tradicionalmente el flujo de información ha sido unidireccional (Palmieri y Rivas, 2007), y sabiendo que el uso estratégico de la información y del conocimiento, garantizan el éxito de los procesos de planificación y desarrollo de instituciones y negocios (Miranda, 1996); uno de los ámbitos que ha experimentado cambios más rápidos y sustanciales en los últimos años es el de la información; este ámbito incluye a los individuos, las organizaciones y las tecnologías de información y comunicación (TIC) (Palmieri y Rivas, 2007); es preciso sin embargo, partir de que la información es por lo tanto una sustancia representativa, y se pueden nombrar ciertas posturas sobre ella: la posesión, la acumulación, exceso ("sobrecarga"), la desigualdad distributiva ("los que tienen y los que no"), la medición (Shannon y Weaver, 1949), la "comoditización" (Schiller, 1994), y así sucesivamente, antes de entender per se el uso de los sistemas de información (Agre, 1995) máxime si todavía el sistema es visto tanto como una cosa y como un agregado de cosas, que posee propiedades emergentes (Wand, Monarchi, Parsons, y Woo, 1995) susceptibles de analizarse en conjunto o por separado, hecho este que en ocasiones se hace desde visiones cercanas, por ejemplo, a la administración y en otras tantas, a disciplinas totalmente diversas y multidimensionales (Bravo, 1994).

Desde el surgimiento de la computación, los sistemas de información (SI) y las tecnologías asociadas han cambiado en forma significativa, quizá como ninguna otra tecnología en la historia, la forma como las empresas y las personas organizan, dirigen, controlan y planean sus negocios (Avison y Pries-Heje, 2005) y con el enorme avance experimentado por las Tecnologías de la Información (TI) en estos últimos años, la capacidad existente para capturar, procesar, almacenar y distribuir la información se ha incrementado de manera espectacular y se han eliminado las barreras espaciales y temporales que en muchos casos dificultaban la coordinación entre las distintas funciones de la empresa (Vieites y Rey, 2008); además de lo anterior descrito, la principal fuerza de campo para los SI, está basada en su capacidad de respuesta a una gran variedad de temas emergentes en las organizaciones a medida que aprenden a explotar el nuevo potencial tecnológico, aunque este aparte esta hasta ahora principalmente orientado a la cuestión y no a la teoría, de modo que los SI carecen del carácter distintivo y del rigor normalmente asociados con las disciplinas científicas y sigue siendo institucionalmente débil (Avgerou, 2000).

Es incuestionable el hecho de que mucha gente está derivando grandes ventajas de estas nuevas fuentes de conocimiento prestadas por redes y sistemas de información, en el marco de lo que ahora se convenciona llamar el sector cuaternario de la economía, responsa- 
ble hoy por los servicios y empleos que dinamizan la tercera ola de las sociedades post-industriales (Miranda, 1996), además de existir una serie de supuestos explícitos e implícitos acerca de la naturaleza de las organizaciones humanas, la naturaleza de la tarea de diseño, y lo que se espera de ellos (Hirschheim y Klein, 1989). Existen herramientas informáticas que hacen de la gestión de la información un asunto más sencillo, rápido y que genera valor mediante el uso de sistemas computarizados (Alvarez y Nuthall, 2006; Lewis, 1998) y que una vez se ha hecho el análisis de la información, lo que permite establecer un diagnóstico, que se utiliza para el establecimiento de metas y objetivos en un próximo periodo productivo y la correspondiente programación operativa. Una vez finalizado el proceso de planificación se procede a su ejecución, mediante la toma de decisiones en el uso de los recursos previstos (García, Acero, y Perea, 2007)

\section{Definiciones}

La situación actual sobre el significado de la palabra información es la inversa, en lugar de haber distintos términos que se refieren a lo mismo, hay un único término, información, que se refiere a fenómenos distintos (Sesé 2007).

El concepto de información aparece en la literatura generalmente vinculado bien con el de datos o bien con el de conocimiento (con la precisión en la definición que la información es un flujo de mensajes mientras que el conocimiento se crea precisamente mediante este flujo de información (Nonaka y Byosiere, 2000); respecto al primero, se establece una relación tan profunda entre ambos vocablos que un gran número de definiciones del término información incluyen el concepto de datos como materia prima o punto de partida de la información, y como producto acabado (Davis y Olson, 1987) lo que supone que la importancia que se concede a la información se debe a su función clave como minimizador de incertidumbre en el proceso de toma de decisiones (Pérez, 2005) y que hace parte de procesos de comunicación social (Gauchi, 2012).

El conocimiento y la información llegan a convertirse en recursos estratégicos y transformadores de la sociedad, igual que el capital y el trabajo lo han sido en la sociedad industrial (Bravo, 1994; Rodríguez 2008) si se usan los datos para su procesamiento y la generación de información de modo que este procesamiento permita mejorar la toma de decisiones, a la vez que se pueda contextualizar la información, clasificar la información, pues define los parámetros de análisis y sistematización, y la categorización de la información, a través de la determinación de las categorías que re- cuperan la información, y que fundamentan la interpretación para su uso posterior (Penagos, Becerra, y Carrillo, 2004).

El concepto de información, lleva una cierta connotación de neutralidad (es homogénea y no controversial), aun cuando en la realidad, es más complicado (Agre, 1995), ya que se define la información y como los datos (en forma de relevante colección de hechos y datos por sí mismos (Font, 2002; Rodríguez 2008) han sido convertidos dentro de un contexto significativo y útil para usuarios finales específicos ( $\mathrm{O}^{\prime}$ Brien, 1999) y que al ser seleccionados y ordenados tanto internos como externos, que se emplean para tomar decisiones respecto a los sistemas (Espinosa, 2014) haciendo que, el enfoque de campo se hubiese ampliando para incluir temas como como fundamentos filosóficos de la información y la comunicación junto con los usos inter-organizacionales de la TI, la naturaleza, uso e impacto de Internet, y aspectos políticos sobre la «sociedad de la información» (Avgerou, 2000).

Se debe partir sin embargo, de los cuatro tipos de conocimientos relacionados a los sistemas de información en desarrollo: el mundo/sujeto (es decir, el dominio representado), el mundo del uso (es decir, el entorno en el que el sistema está siendo utilizado), el mundo del desarrollo (es decir, el proceso y el medio ambiente en el que el sistema es desarrollado), y el mundo del sistema (es decir, la información del sistema mismo (Wand et al., 1995).

En líneas similares, (Drucker, 1989) se establece que la información son datos que tienen una relevancia y un propósito y (Emery, 1990) puntualiza que la información se deriva de los datos con la intención de hacerla disponible para el decisor. Por tanto, para alcanzar esa relevancia o propósito para el decisor, los datos o materia prima requieren un proceso de transformación, lo que resalta que la información no consiste en datos simples, sino procesados de alguna forma; por ejemplo, ordenados y resumidos, para proporcionar un resultado, interpretado como información por el usuario o personal encargado de la toma de decisiones (Lucas, Díaz, y Quintero, 2000). Este proceso de transformación exige dotar a los datos de una forma determinada y de un significado mediante intervención humana (Laudon y Laudon, 2000; Pérez, 2005), usando por ejemplo la construcción de modelos.

Los modelos son bases de datos estructuradas, que ordenan la información disponible sobre cierto sistema y permiten contestar preguntas muy específicas. Son una manera de ordenar, transmitir y utilizar la información técnica y científica; así, un sistema de información 
se define como un conjunto de procedimientos interrelacionados que forman un todo, es decir, obtiene, procesa, almacena y distribuye información (datos manipulados) para apoyar la toma de decisiones y el control en una organización (Espinosa, 2014), que engloba a personas, máquinas y/o métodos organizados con la infraestructura, la organización, el personal y todos los componentes necesarios para la recopilación, procesamiento, almacenamiento, transmisión, visualización, diseminación y organización de la información (Berzal, 2004), sobre la claridad conceptual descrita de la existencia de cinco etapas para la captura de los datos, que van desde los datos sobre papel hasta las bases de datos relacionales $y$, últimamente, las bases multimedia, que almacenan también imágenes, sonidos y cuantos objetos defina el diseñador (Gray, 1996).

Derivándose de lo anterior se puede inferir que un sistema de información es un conjunto formal de procesos que operado sobre una colección de datos estructurados de acuerdo a las necesidades de la empresa, recopila, elabora y distribuyen selectivamente la información necesaria para la operación de dicha empresa y para las actividades de dirección y control correspondientes, apoyando al menos en parte, los procesos de toma de decisiones necesarios para desempeñar funciones de negocio de la empresa de acuerdo con su estrategia (Andreu, Ricart, y Valor, 1991) que complementa y apoya la toma de decisiones, el control, análisis y visión de una organización (Hernández, 1997). Otra definición sugiere el sistema de información como el conjunto de personas, datos, información, herramientas de procesamiento y almacenamiento de información dentro de una organización a través de las Tecnologías de la Información y Comunicación (Heeks, 1998).

Respecto de los tipos de sistemas de información se pueden clasificar según sus tipologías en:

\begin{tabular}{|l|l|}
\hline $\begin{array}{c}\text { Tipo de Sistema de } \\
\text { Información }\end{array}$ & \multicolumn{1}{|c|}{ Tipos } \\
\hline Grado de formalidad & $\cdot \begin{array}{l}\text { Formales } \\
\text { Informales }\end{array}$ \\
\hline Automatización & $\cdot \begin{array}{l}\text { Manuales } \\
\text { Informáticos }\end{array}$ \\
\hline $\begin{array}{l}\text { Relación con la } \\
\text { toma de decisiones }\end{array}$ & $\begin{array}{l}\text { Estratégicos (alta dirección) } \\
\text { Gerencial (nivel intermedio) }\end{array}$ \\
\hline
\end{tabular}

\begin{tabular}{|c|c|}
\hline Funcionalidad & $\begin{array}{ll}\cdot & \text { Gestión comercial } \\
\text { - } & \text { Gestión contable } \\
\text { - } & \text { Gestión financiera } \\
\text { - } & \text { Gestión de Recursos Humanos de la Producción }\end{array}$ \\
\hline $\begin{array}{l}\text { Grado } \\
\text { Especialización }\end{array}$ & $\begin{array}{l}\text { - Específicos } \\
\text { - Generales }\end{array}$ \\
\hline
\end{tabular}

Fuente: Adaptado de (Bravo, 1999; Edwards, Ward, y Bytheway, 1998)

También se referencian los sistemas de información, dependiendo de las necesidades de los usuarios humanos y la empresa. Los sistemas de procesamiento de transacciones (TPS) funcionan en el nivel operacional de la organización; los sistemas de automatización de oficinas (OAS) y los sistemas de trabajo de conocimiento (KWS) brindan soporte para el trabajo a nivel del conocimiento. Entre los sistemas de nivel superior se encuentran los sistemas de información administrativa (MIS) y los sistemas de soporte de decisiones (DSS) (Kendall y Kendall, 2005).

Como gran conclusión, los datos, información y el conocimiento son eslabones de una cadena cíclica en la que el último eslabón realimenta la cadena, a la vez que el valor del recurso y la aportación e implicación humana son cada vez mayores (Pérez, 2005).

\section{Usos}

Para obtener un adecuado sistema informático en cualquier tipo, tamaño y diseño de una organización económica y social, sea una empresa, sea un organismo institucional con finalidad de obtención de beneficio o de ofrecer un servicio, es necesario analizar, diseñar y construir previamente un Sistema de Información (SI) (Rincón y Sanz, 2000); la información es clave también al nivel de los beneficiarios; el desarrollo está relacionado con el empoderamiento $y$, a pesar de que habitualmente existen situaciones de dominancia o subordinación, los beneficiarios deben tener información para tener libertad de elegir, decidir, opinar o comportarse de una determinada manera (Baumann, 2000).

Todo conocimiento y saber es una interpretación; sin embargo, la interpretación de la realidad no es posible sin teoría, pues el secreto es descubrir lo que hay tras la información (Penagos et al., 2004) y como varios estudios lo muestran, la adopción y uso de las tecnologías de la información (TI), tiende a aumentar la rentabilidad esperada dependiente de este uso; esto intuitivamente tiene sentido, ya que se podría suponer 
que los agricultores pudieran maximizar sus beneficios (Kuhlmann y Brodersen, 2001) con un uso adecuado de los mismos.

El principal uso de la información es no ser consumible, pues quien la entrega o comparte no la pierde e incluso puede ser utilizada simultáneamente por varios individuos; es transmisible, acumulativa y aumentable con costos marginales inapreciables; es por tanto sinergética, no desgastándose con su uso sino perfeccionándose (Pérez, 2005), de ahí que una buena gestión es una condición "sine qua non" para aumentar la eficiencia y eficacia de un sistema determinado (Font, 2002); la información es el ingrediente básico de cualquier innovación tecnológica y un elemento clave en todo proceso de innovación o de investigación (Palmieri y Rivas, 2007). Los sistemas de gestión empresarial se limitaron en sus orígenes a almacenar solamente los datos procedentes de las operaciones de las empresas, estructurados en bases de datos relacionales (Vieites y Rey, 2008), desconociendo que la información se ha convertido de hecho en un verdadero recurso estratégico denominado como el principal activo invisible de las empresas (Bravo, 1994) y que paralelamente se necesita conocer la estrategia de la organización y el tipo de organización para posteriormente establecer las necesidades de información y adquirir las herramientas necesarias para el desarrollo del sistema de información (Hernández, 1997), de manera que se pueda omitir la difusión lenta, que tiende a tener un efecto sobre el desempeño de la organización comparado con la adquisición de información (Fichman y Kemerer, 1999)

Otro de los aspectos importantes es que la información requiere dos componentes principales: herramientas informáticas y habilidades de información. Las herramientas pueden ayudar, pero sólo después de haber discriminado qué información interesa entre toda la disponible. Ciertamente, la selección debe ser realizada todavía por el ser humano, quién también deberá saber cómo aplicarla a la resolución de problemas, al aprendizaje o la toma de decisiones (Pítsica, 2004).

Los desafíos incluyen la necesidad de incorporar la información de forma explícita dentro de la planificación y ejecución de cualquier proceso institucional y aprovechar al máximo las posibilidades que ofrecen las Tecnologías de la información y comunicación (TIC) disponibles (Palmieri y Rivas, 2007) ya que la información aumenta la posibilidad de toma de decisiones correctas (Font, 2002), hasta llegar a niveles de certeza donde no habría equívoco posible (Ciampagna, 2000), a través del uso de las herramientas del pensamiento sistémico como son los modelos y de particular forma poder transformar un sistema de información en un sistema de apoyo a la decisión empresarial para el productor ovino, de modo que las tecnologías y sistemas de información permitan a la empresa obtener mejores resultados que sus competidores, constituyéndose por sí mismas como una fuente de ventaja competitiva y una poderosa arma que permita diferenciarse de sus competidores y obtener mejores resultados que estos. De este modo los sistemas de información se constituyen como una de las cuestiones estratégicas de la empresa, que ha de considerarse siempre en todo proceso de planificación empresarial (Hernández, 1997)

Actualmente el universo está caracterizado por la abundancia de datos (information overload) y poca objetividad en términos de información segmentada (Miranda, 1996), siendo necesario retomar el enfoque de la sociedad del conocimiento que va a enaltecer la información como elemento esencial de la actividad empresarial y a establecer nuevas formas de entender y desarrollarse a sí misma (Bravo, 1994) de manera que la información se convierta en un elemento clave para la gestión, así como para la supervivencia y crecimiento de la organización empresarial (Hernández, 1997).

Los actores y agentes del desarrollo son ricos en cuanto a la información que obtienen, esta información debe ser procesada en el diseño y planificación de programas y en los posibles cambios que van aconteciendo. La gestión de la información significa descubrir qué información es necesaria para la gente con la que se trabaja, de dónde debería proceder y para lo que sería necesaria; lo que significa comprender la información como un recurso disponible para la organización y que puede ser utilizado de forma consciente para satisfacer sus necesidades (Rodríguez 2008); ahí sobresale que la importancia que se concede a la información desde su función clave como minimizador de incertidumbre (Drucker, 1989) en el proceso de toma de decisiones, al cual se considera como definitorio de la labor del directivo, sea cual sea su nivel jerárquico (Turban, McLean, y Wetherbe, 2001) que por demás presta atención al contexto social y a la interacción entre grupos, individuos y sistemas, la información se aprecia desde posiciones interpretativas e históricas, donde la percepción se encuentra afectada por factores culturales (Gauchi, 2012).

\section{Limitantes al caso ovino colombiano}

A nivel de una organización existe información formal e informal que puede reconocerse como recurso económico fundamental, junto a los ya considerados por la teoría económica tradicional: tierra, trabajo y capital (Boland y Hirschheim, 1992). 
La necesidad de información es tan amplia que se procuran sistemas de información de alta complejidad y variada calidad, a todo nivel de las decisiones empresariales (Ciampagna, 2000), siendo indispensable tener presente (y con mayor detalle para el caso ovino colombiano) requerimientos de factibilidad técnica y económica, integración, interfase usuario/sistema, fuerzas de la competencia, calidad de la información y disponibilidad, requerimientos del sistema per se, requerimientos de procesamientos de datos, requerimientos de costo/beneficio y el más importante el factor humano (Espinosa, 2014), de modo que se cumplan premisas visionarias del siglo pasado respecto de "seres para quienes no existen las distancias, con enorme capacidad para manejar información y analizar sistemas que por su distancia, escala o complejidad escapan a las posibilidades del mortal común (De Rosnay, 1995)".

La gestión del conocimiento, la explotación inteligente del capital intelectual, el desarrollo de los mercados internos de conocimientos y el estímulo de la creatividad son quizás más importantes que la pura gestión de las tecnologías de la información. Las tecnologías no consiguen su efecto sin personas que sepan utilizarlas adecuadamente, para conseguir sacar rendimiento a la inversión en sistemas (Pítsica, 2004).

El producto final del proceso, que casi siempre incluirá el diseño y prueba de alternativas, es información para innovar en el proceso productivo (Palmieri y Rivas, 2007); las actividades de control de los procesos productivos, de identificación de limitantes y de planificación difícilmente se llevan a cabo exitosamente si el gerente (llámese productor, administrador, asesor, etc.) no cuenta con la información pertinente (González, Grajales, Manrique, y Téllez, 2011), toda vez que los sistemas de información los que habilitan a la organización a resolver los problemas y/o son parte de la solución de los mismos (Ciampagna, 2000) como el uso de las tecnologías de la información TI (p.e. la computadora) no redundan actualmente en mejores y más rápidas decisiones y, no ayudan en este momento en menores costos de producción y por ende no tienen un efecto marcado sobre la economía.

Actualmente se pone más énfasis en el flujo de la información que en su uso efectivo, probablemente porque hay una creencia generalizada de que las estructuras organizacionales modernas se validan por su capacidad de ofrecer condiciones para el proceso de toma de decisiones, en un ambiente competitivo, sin cuestionar si hay barreras en la transferencia de información (Miranda, 1996), desconociendo que el papel de un modelo conceptual (en este caso de un sistema de información para los productores ovinos de Colombia) también puede ser escrito desde el punto de vista del desarrollo de sistemas que consiste en el análisis, diseño e implementación; el análisis transforma un mundo real percibido a través de un sistema en un modelo conceptual de ese sistema; el diseño transforma el modelo conceptual de la situación objeto de estudio, en un modelo de sistema de información y la implementación transforma el modelo del sistema de información un sistema de información funcional, que es una representación ejecutable por máquina (Wand et al., 1995) en los que se requiere evidenciar la interactividad y flexibilidad de los sistemas de información como un punto clave en el éxito o fracaso (Hernández, 1997), asumiendo que la teoría permitiría, en cierto modo, mirar los hechos, organizarlos y representarlos conceptualmente, pues facilita la organización de la información y hace explícita la simultaneidad (Penagos et al., 2004) a pesar del continuo avance tecnológico en materia informática y de telecomunicaciones, lejos de incrementar el valor de la información lo ha disminuido (Pérez, 2005)

La posesión o privación del derecho a la información, configurarían el imperialismo informacional (information imperialism) que puede ampliar, aunque relativamente, las odiosas distancias entre grupos sociales y hasta regiones (Miranda, 1996) (incluido el renglón pecuario nacional y no escapando la ovinocultura); se quieren aumentar la eficiencia económica de la producción y los procesos de comercialización siendo necesario desarrollar y perfeccionar el conocimiento basado en sistemas de apoyo a las decisiones (por sus siglas en inglés DSS) (Kuhlmann y Brodersen, 2001) para evitar el reforzamiento de las ya existentes brechas sociales y por qué no mencionarlo, de la brecha digital como reflejo de otras brechas sociales y económicas, pero también es en sí misma una causa de exclusión que retroalimenta a las otras brechas ya existentes, y contribuye a acrecentar las disparidades (Prat, 2004).

El logro de los objetivos básicos de las organizaciones que promueven la innovación tecnológica agropecuaria, como su propia sostenibilidad, dependen de la entrega de información pertinente y por medios adecuados a diferentes públicos (Palmieri y Rivas, 2007) siendo indispensable entender que un sistema de información abarca más que el aspecto meramente computacional, pues no sólo se debe tener en cuenta estas herramientas, sino también el modo de organizar dichas herramientas y de obtener la información necesaria para el correcto funcionamiento (Hernández, 1997) de la agroempresa ovina colombiana. 
Cambiar la forma de reagrupar la información, de procesarla y usarla para la toma de decisiones implica modificar la propia estructura de la organización (Helfenbein, Sawyer, Sayer, y Wijesinghe, 1987) de forma que los sistemas de información constituyan uno de los aspectos estratégicos claves para el buen hacer de la empresa siendo necesario que la totalidad de la organización (sector ovino colombiano) esté concienciado de su utilidad al realizar el proceso de planificación estratégica de la empresa. Ha de existir una política de información y motivación dentro de la empresa, en la que por ejemplo, la formación y la educación futura, a nivel de los dueños de las unidades productivas usen las herramientas disponibles en el mercado deben tener una alta prioridad asegurando que los agricultores sepan qué datos se están utilizando (Kuhlmann y Brodersen, 2001), el origen de los datos y por supuesto su importancia dentro de la producción.

\section{Conclusiones}

El verdadero reto para las organizaciones (para el caso las agropecuarias) consiste en transformar la documentación y la información en un recurso estratégico que facilite la toma de decisiones, se dote de capacidad de reacción ante los cambios en el entorno, permita descubrir nuevas oportunidades de negocio y aumente la eficacia de la gestión empresarial (Gauchi, 2012) para que, la gente pueda tener suficiente información para tomar sus decisiones y, segundo, que su voz y puntos de vista sean escuchados y valorados (Powell, 2003). Los marcos de referencia para el ámbito de los $\mathrm{SI}$, suelen orientarse a modelos estructurales complejos, nada adecuados para la gestión por competencias (Urquiza, 2007).

El sistema de información puede ser técnicamente perfecto, pero su valor es cuestionable si no es usado. Los sistemas de información que no contribuyan, por ejemplo, a un mejor proceso de toma de decisiones que lleve a su vez a mejores resultados por parte de la organización, son sistemas informalmente estériles (Pítsica, 2004). El estudio de los efectos de la adopción de TIC (como herramienta del SI) en distintos sectores de actividad económica, ha estado generalmente asociado a la medición de su impacto sobre la tasa de productividad sectorial, y más específicamente, sobre la variación del componente residual conocido como "productividad total de factores" (PTF) (Ministerio de Asuntos Exteriores y de Cooperación, 2010)

Debe evolucionarse hacia el uso de la planificación estratégica de los sistemas de información (por sus siglas en inglés SISP), de modo que se puedan identificar las relaciones entre los sistemas de información (SI) y el uso de la Tecnologías de la información (TI), aún y cuando actualmente siga siendo más dominante la relación $\mathrm{SI} / \mathrm{TI}$ en el proceso de planeación estratégica (Hernández, 1997; Silvius y Stoop, 2013), todo enmarcado en que el dinámico ambiente de negocios global de hoy ha evolucionado las $\mathrm{TI}$, hasta convertirlas en una herramienta para vincular la mayoría de las facetas de la operaciones de negocios de los proveedores a través de la cadena de suministro y operaciones de negocio a los consumidores (Wilkin y Cerpa, 2012), haciendo que los sistemas estratégicos de información permitan a la empresa sobrevivir en entornos altamente competitivos y lograr un crecimiento de la organización (Hernández, 1997), con la claridad que si bien información e informática se interrelacionan, hay que dejar claro que dentro del vínculo que se da entre ambos, el concepto más relevante es el de información (González 1994)

La TI necesita tener un SI definido previo para cumplir su objetivo; para su utilización sin esta base previa, es muy probable que lleve al fracaso o en el mejor de los casos a la sub-utilización de los medios tecnológicos (Ciampagna, 2000), tal cual sucede en el sector agropecuario colombiano, que además requiere crear, preparar e implementar productos y servicios de información cualitativamente superiores, que satisfagan del todo las necesidades informacionales de los productores campesinos.

El proceso de desarrollo de los sistemas de información afectará en gran medida al éxito o fracaso de la organización; las organizaciones tendrán que adecuar los sistemas de información a sus recursos de capital y las necesidades de la organización; toda empresa/ organización ha de considerar los sistemas de información como un todo, un elemento más de su política de negocio (Hernández, 1997).

Debido a que existe un conocimiento imperfecto (en la actividad pecuaria y ovina colombianas) en cuanto a las relaciones entrada/salida de los sistemas de producción, así como de las variables incontrolables climático-espaciales, se hace necesaria una estrecha cooperación con las disciplinas biológicas de las ciencias agropecuarias. Es importante orientar la investigación hacia el usuario final con enfoque multidisciplinario como factor clave para seguir avanzando (Kuhlmann y Brodersen, 2001), de manera que los Si puedan ser en el mediano plazo, elementos que permitan el fortalecimiento y posicionamiento de la cadena ovina colombiana, como modelo de susbistencia eficiente para pequeños productores y como jalonar de ingresos para el sector pecuario nacional. 
Como reflexión de cierre, Mario Bounge, filósofo argentino, define que "la información en sí misma no vale nada, hay que descifrarla. Hay que transformar las señales y los mensajes auditivos, visuales o como fueren, en ideas y procesos cerebrales, lo que supone entenderlos y evaluarlos. No basta poseer un cúmulo de información. Es preciso saber si las fuentes de información son puras o contaminadas, si la información como tal es fidedigna, nueva y original, pertinente o impertinente a nuestros intereses, si es verdadera o fal$\mathrm{sa}$, si suscita nuevas investigaciones o es tediosa y no sirve para nada, si es puramente conceptual o artística, si nos permite diseñar actos y ejecutarlos o si nos lo impide. Mientras no se sepa todo eso, la información no es conocimiento".

\section{Agradecimientos}

El presente documento surge del macro-proyecto "Desarrollo e implementación de un Sistema de Gestión Tecnológica en los sistemas de producción de la cadena ovino - caprina para el mejoramiento de su competitividad (SIGETEC)", codificado por el Ministerio de Agricultura y Desarrollo Rural con el número 2007Q6350-162 y con código interno QUIPU: Código MADR 068-2007Q6350-162, adscrito a la facultad de medicina veterinaria y de zootecnia de la Universidad Nacional de Colombia y desarrollado particularmente con la tesis doctoral titulada "Prácticas ganaderas en sistemas de producción ovinos: desafíos para el mejoramiento de la competitividad del sector en Colombia".

\section{Referencias}

Agre PE. Institutional circuitry: Thinking about the forms and uses of information. Information Technology and Libraries. 1995;14(4):225-230.

Alvarez J, Nuthall P. Adoption of computer based information systems: The case of dairy farmers in Canterbury, NZ, and Florida, Uruguay. Computers and Electronics in Agriculture. 2006;50(1):48-60.

Andreu R, Ricart J, Valor J. Estrategia y sistema de información: McGraw-Hill.

Avgerou, C. (2000). Information systems: what sort of science is it? Omega, 1991;28(5):567-579. doi:http://dx.doi.org/10.1016/ S0305-0483(99)00072-9

Avison DE, Pries-Heje J. 2005. Research in information systems: A handbook for research supervisors and their students (G. P. Publishing Ed.): Elsevier Butterworht- Heinemann.

Baumann P. 2000. Sustainable livelihoods and political capital: Arguments and evidence from decentralisation and natural resource management in India: Overseas Development Institute.
Berzal F. 2004. El ciclo de vida de un sistema de información. Retrieved from http://elvex.ugr.es/

Boland RJ, Hirschheim RA. 1992. Critical issues in information systems research: John Wiley \& Sons, Inc.

Bravo DG. 1994. Sistemas y tecnologías de la información en las organizaciones. Repercusiones para la administración. (Doctoral dissertation), Universidad de Alicante, España.

Bravo DG. 1999. Sistemas de información en la empresa: conceptos y aplicaciones: Ediciones Pirámide.

Ciampagna JM. 2000. Los sistemas de información en la organización. Retrieved from https://elprofejose.files.wordpress. com/2011/10/03_los-sistemas-de-informacic3b3n-en-la-organizacion.pdf

Davis G, Olson M. 1987. Sistemas de Información Gerencial. Colombia: McGraw-Hill.

De Rosnay J. 1995. L'homme symbiotique: regards sur le troisième millénaire. Paris: Editions du Seuil.

Drucker PF. 1989. Las Nuevas Realidades. Barcelona: EDHASA.

Edwards C, Ward J, Bytheway A. 1998. Fundamentos de sistemas de información. Madrid: Prentice Hall.

Emery JC. 1990. Sistemas de Información para la Dirección. El Recurso Estratégico Crítico. Madrid: Díaz de Santos.

Espinosa F. 2014. Gestión del mantenimiento Retrieved from http:// campuscurico.utalca.cl/ fespinos/

Fichman R, Kemerer C. The Illusory Diffusion of Innovation: An Examination of Assimilation Gaps. Information Systems Research. 1999;10(3):255-275.

Font F. 2002. Evaluación del sistema de información de salud en el distrito de Kilombero, Tanzania. (Tesis Doctoral), Universitat de Barcelona.

García A, Acero R, Perea J. 2007. Libro virtual de economía y gestión. Documentos de trabajo UCO-6, (4). España.

Gauchi V. Aproximación teórica a la relación entre los términos gestión documental, gestión de información y gestión del conocimiento. Revista Española de Documentación Científica. 2012;35(4): 531-554.

González C, Grajales H, Manrique C, Téllez G. Gestión de la información en los sistemas de producción animal: -una mirada al caso de la ovino-caprinocultura. Revista de la Facultad de Medicina Veterinaria y de Zootecnia. 2011;58:176-193.

González M. 1994. Implicaciones estratégicas de los sistemas y tecnologías de la información: el caso de la provincia alicantina. (Tesis Doctoral), Universidad de Alicante.

Gray J. Data Management: Past, Present, and Future. IEEE Computer, 1996;29(10):38-46.

Heeks R. 1998. Information technology and public sector corruption: Institute for Development Policy and Management, University of Manchester. 
Helfenbein S, Sawyer H, Sayer P, Wijesinghe S. 1987. Technologies for management information systems in primary health care. Washington, D.C: World Federation of Public Health Associations.

Hernández A. Los sistemas de información: evolución y desarroIlo. Proyecto social: Revista de relaciones laborales. 1997;1011:149-165.

Hirschheim R, Klein HK. Four paradigms of information systems development. Communications of the ACM. 1989;32(10):11991216.

Kendall KE, Kendall JE. 2005 Análisis y diseño de sistemas: Pearson educacion.

Kuhlmann F, Brodersen C. Information technology and farm management: developments and perspectives. Computers and Electronics in Agriculture. 2001;30(1):71-83.

Laudon KC, Laudon JP. 2000. Management Information Systems: New Approaches to Organization and Technology: Prentice Hall PTR.

Lewis T. Evolution of farm management information systems. Computers and Electronics in Agriculture. 1998;19(3):233-248.

Lucas H, Díaz D, Quintero L. 2000. La tecnología de la información y la paradoja de la productividad: cómo evaluar el valor de las inversiones en tecnología de la información: Oxford University Press.

Ministerio de Asuntos Exteriores y de Cooperación. 2010. Tecnologías de la Información y Comunicaciones (TIC) en la Gestión Ganadera. Contexto y vectores que las propician. Madrid, España: AHCIET.

Miranda A. Globalización y sistemas de información: nuevos paradigmas y nuevos desafíos. Ciência da Informação. 1996;25(3):.

Nonaka I, Byosiere P. 2000. La creación de conocimiento regional: un proceso de desarrollo social. Cluster conocimiento. Las sociedades del conocimiento. Bilbao.

O’Brien J. 1999. Managing Information Technology in the Internet Worked Enterprise (4 ed.): McGraw Hill.

Palmieri V, Rivas L. Gestión de información para la innovación tecnológica agropecuaria. COMUNIICA. 2007;3:17-25.

Penagos R, Becerra A, Carrillo A. 2004. La práctica investigativa en ciencias sociales: Universidad Pedagógica Nacional.

Pérez D. 2005. Contribución de las tecnologías de la información a la generación de valor en las organizaciones: un modelo de análisis y valoración desde la gestión del conocimiento, la productividad y la excelencia en la gestión. tesis doctoral], Santander, Universidad de Cantabria.

Pítsica M. 2004. Sistema de información para la gestión aplicado en las entidades financieras: estudio empírico Santa Catarina.
(Tesis Doctoral), Universidad Complutense de Madrid, Servicio de Publicaciones.

Powell M. 2003. Information management for development organisations (Second ed.): Oxfam Development Guidelines.

Prat J. La gestión de la información vía Internet como factor de la reducción de la brecha digital entre países europeos e iberoamericanos. Documentación de las ciencias de la información 2004; (27): 43-52.

Rincón J, Sanz I. 2000. Evaluación de los sistemas de información y comunicación (SIC). Paper presented at the Anales de Economía Aplicada. XIV Reunión ASEPELT, Oviedo, España

Rodríguez C. 2008. Sistemas de información basados en indicadores de seguimiento de proyectos en organizaciones de cooperación descentralizada. Estudio de caso de los proyectos cofinanciados por la Comunidad de Madrid en Perú. (Tesis doctoral), Universidad Politécnica de Madrid., España.

Schiller D. From culture to information and back again: Commoditization as a route to knowledge. Critical Studies in Mass Communication. 1994:1:92-92.

Sesé F. 2007. Propuesta de un método de validación de esquemas conceptuales y análisis comparativo de la noción de información en los métodos de desarrollo de sistemas de información. (Tesis Doctoral), Universitat Ramon Llull, Barcelona, España.

Shannon C, Weaver W. 1949. The mathematical theory of communication (Urbana, IL: University of Illinois Press IL.

Silvius A, Stoop J. The Relationship between Strategic Information Systems Planning Situational Factors, Process Configuration and Success. Journal of International Technology and Information Management. 2013;22(1):1.

Stiroh K. Is there a new economy? Challenge. 1999;42(4):82-101.

Turban E, McLean E, Wetherbe J. 2001. Information Technology for Strategic Advantatge. New York: John Wiley \& Sons, 2nd edition.

Urquiza A. Aplicación de modelos de competencias a la gestión de sistemas de información. Revista Española de Innovación, Calidad e Ingeniería del Software, 2007;3(1):

Vieites A, Rey C. 2008. Sistemas de información. Herramientas prácticas para la gestión empresarial. RAMA.

Wand Y, Monarchi D, Parsons J, Woo C. Theoretical foundations for conceptual modelling in information systems development. Decision Support Systems, 1995;15(4):285-304. doi:http://dx.doi. org/10.1016/0167-9236(94)00043-6

Wilkin C, Cerpa N. Strategic Information Systems Planning: An Empirical Evaluation of Its Dimensions. Journal of technology management \& innovation, 2012;7:52-62. 